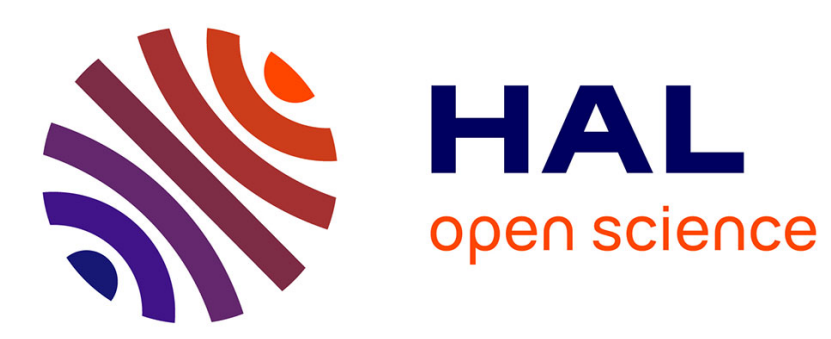

\title{
More precise tracking of horizontal than vertical target motion with both the eyes and hand
}

Frederic S Danion, James Mathew, Niels Gouirand, Eli Brenner

\section{To cite this version:}

Frederic S Danion, James Mathew, Niels Gouirand, Eli Brenner. More precise tracking of horizontal than vertical target motion with both the eyes and hand. Cortex, 2021, 134, pp.30-42. 10.1016/j.cortex.2020.10.001 . hal-03024788

\section{HAL Id: hal-03024788 \\ https://hal.science/hal-03024788}

Submitted on 11 May 2021

HAL is a multi-disciplinary open access archive for the deposit and dissemination of scientific research documents, whether they are published or not. The documents may come from teaching and research institutions in France or abroad, or from public or private research centers.
L'archive ouverte pluridisciplinaire HAL, est destinée au dépôt et à la diffusion de documents scientifiques de niveau recherche, publiés ou non, émanant des établissements d'enseignement et de recherche français ou étrangers, des laboratoires publics ou privés. 


\title{
More precise tracking of horizontal than vertical target motion with both the eyes and hand
}

\author{
Frederic R. Danion ${ }^{1}$, James Mathew ${ }^{1,2}$, Niels Gouirand ${ }^{1}$, and Eli Brenner ${ }^{3}$ \\ ${ }^{1}$ Aix Marseille Université, CNRS, Institut de Neurosciences de la Timone UMR 7289, \\ Marseille, France \\ ${ }^{2}$ current affiliation: Institute of Neuroscience, Institute of Communication \& Information \\ Technologies, Electronics \& Applied Mathematics, Université Catholique de Louvain, \\ Belgium
}

${ }^{3}$ Department of Human Movement Sciences, Vrije Universiteit, Amsterdam, the Netherlands

\begin{abstract}
When tracking targets moving in various directions with one's eyes, horizontal components of pursuit are more precise than vertical ones. Is this because horizontal target motion is predicted better or because horizontal movements of the eyes are controlled more precisely? When tracking a visual target with the hand, the eyes also track the target. We investigated whether the directional asymmetries that have been found during isolated eye movements are also present during such manual tracking, and if so, whether individual participants' asymmetry in eye movements is accompanied by a similar asymmetry in hand movements. We examined the data of 62 participants who used a joystick to track a visual target with a cursor. The target followed a smooth but unpredictable trajectory in two dimensions. Both the mean gaze-target distance and the mean cursor-target distance were about $20 \%$ larger in the vertical direction than in the horizontal direction. Gaze and cursor both followed the target with a slightly longer delay in the vertical than in the horizontal direction, irrespective of the target's trajectory. The delays of gaze and cursor were correlated, as were their errors in tracking the target. Gaze clearly followed the target rather than the cursor, so the asymmetry in both eye and hand movements presumably results from better predictions of the target's horizontal than of its vertical motion.
\end{abstract}




\section{1-Introduction}

People direct their gaze at important objects. Doing so presumably helps prevent collisions with them if they are obstacles and helps one to reach them if the goal is for instance to grasp them (Johansson et al., 2001). If such objects or the observer is moving, or both are moving, the eyes move to maintain gaze on the object of interest (Barnes, 2008; Spering et al., 2011). Although it is not evident that it is more important to keep the image of a moving object of interest close to the fovea in the horizontal than in the vertical direction, it has repeatedly been shown that horizontal components of pursuit eye movements are more accurate than vertical ones. This has been demonstrated for children (Grönqvist et al., 2006; Robert et al., 2014; Takeichi et al., 2003), adults (Baloh et al., 1988; Collewijn \& Tamminga, 1984; Ke et al., 2013, 2013; Lipton et al., 1980; Rottach et al., 1996), and even cats and monkeys (Evinger \& Fuchs, 1978; Kettner et al., 1996). More precise horizontal tracking has not only been found when motion of the target is restricted to the vertical or horizontal axis (Baloh et al., 1988; Lipton et al., 1980; Robert et al., 2014), it is also observed for the horizontal and vertical components of pursuit of a target that is moving in both dimensions (Ke et al., 2013; Kettner et al., 1996; Soechting et al., 2010). The possibility of having direction-dependent differences in the precision of pursuit is in line with neurophysiological studies arguing for separate neural substrates for horizontal and vertical eye movements (Chubb et al., 1984; Kettner et al., 1996; Saito \& Sugimura, 2020).

Despite all this evidence that horizontal components of pursuit eye movements are more precise than vertical ones, the reason(s) for this directional asymmetry remains largely unknown. It has been suggested that everyday experience may be responsible for this bias, because in daily life objects that are pursued tend to mainly move horizontally (Collewijn \& Tamminga, 1984). Even if this is the case, it is not evident whether the asymmetry arises because horizontal target motion is predicted better or because horizontal movements of the 
eyes are controlled more precisely. To determine which is more likely, we make use of the fact that we know that when tracking a visual target with the hand, the eyes also track the target (Danion \& Flanagan, 2018; Niehorster et al., 2015; Xia \& Barnes, 1999). We investigate whether the directional asymmetry that has been found during isolated eye movements is also present during manual tracking of a target that moves in a haphazard manner across a frontal plane, and if so, whether the asymmetry in eye movements is accompanied by a similar asymmetry in hand movements. We reason that if tracking is more precise in the horizontal than in the vertical direction for both eye and hand movements, the directional asymmetry is likely to result from better prediction of the target's motion along the horizontal axis, rather than from the control of the movement itself independently being more precise for horizontal movements of both the eye and hand. If the directional asymmetry arises from better prediction we also expect tracking errors to be correlated across the effectors. Although prediction is believed to play an important role in many aspects of human behaviour (Bar, 2007; Bubic et al., 2010; Clark, 2013) its role in guiding movements is still being debated (Fiehler et al., 2019; Zhao \& Warren, 2017) as are the neural mechanisms involved (see Hogendoorn, 2020 for a recent overview).

The possibility that eye and hand movements exhibit a similar directional asymmetry is grounded upon the observation that there is an intricate relationship between eye and hand movements in tasks such as reaching (Crawford et al., 2004; Miall et al., 2001; Neggers \& Bekkering, 2000; Prablanc et al., 1979) and intercepting (Mrotek and Soechting 2007; de la Malla Smeets \& Brenner, 2017; but see Cámara et al, 2020), as well as tracking (Bock, 1987; Danion \& Flanagan, 2018; Huang \& Hwang, 2012; Koken \& Erkelens, 1992; Mather \& Putchat, 1983; Miall et al., 2001; Niehorster et al., 2015). In tracking, making eye movements is believed to help guide the hand (Danion \& Flanagan, 2018; Gouirand et al., 2019), but making hand movements is also believed to help keep the eyes on the target (Danion \& 
Flanagan, 2018; Huang \& Hwang, 2012; Koken \& Erkelens, 1992; Mather \& Putchat, 1983; Niehorster et al., 2015), in particular by increasing smooth pursuit gain and thereby decreasing the number of catch-up saccades. There is some evidence that horizontal eye movements are more precise than vertical ones in a manual interception task (Mrotek \& Soechting, 2007), but in that study the asymmetry was observed before the hand started to move, and there was no comparison between the precision of horizontal and vertical hand movements.

Here, we reanalyse the data of previous studies in which a large set of participants were asked to move a cursor with a joystick so that it tracked a visual target that followed a smooth but unpredictable trajectory in the frontal plane (Gouirand et al., 2019; Mathew et al., 2018, 2019). Having simultaneously recorded both eye and hand movements in our previous studies allows us not only to assess the directional asymmetry in precision for each effector, but also to compare this asymmetry across effectors. Since the motion that participants were asked to track is smooth but not completely predictable, we expect participants to try to anticipate the target's future path, but not to be able to do this so well that tracking is almost perfect. This will allow us to quantify differences between the horizontal and vertical lag and precision of movements of both the eye and hand with respect to the target's motion.

\section{2-Methods}

\subsection{Participants}

For the purpose of the current study, we analyzed data from sixty-two right-handed volunteers (31 females; $26.8 \pm 6.2$ years of age; from here on this notation will be used to indicate mean \pm standard deviation). The data are from the initial, identical baseline trials of three separate groups of participants, from three separate experiments (Gouirand et al., 2019; 
Mathew et al., 2018, 2019). All participants gave written consent prior to participation. The experimental paradigm (2016-02-03-007) was approved by the local ethics committee of AixMarseille University and complied with the 2008 Declaration of Helsinki.

\subsection{Data Acquisition}

Seated in a dark room, participants faced a screen (BENQ, 1920×1080, 27" inch, $144 \mathrm{~Hz}$ ) positioned in the frontal plane $57 \mathrm{~cm}$ from their eyes (see Fig. 1A). Head movements were restrained by a chin and forehead rest. In order to prevent vision of the hands, a mask was positioned under the chin. With the right forearm being supported, participants had to hold a joystick (Series 812, Megatron, France, with $\pm 25^{\circ}$ of inclination and no force bringing it back to the central position) that was positioned in line with their central sagittal plane. The output signals of the joystick were fed into a data acquisition system (Keithley ADwin RealTime, Tektronix) and sampled at $1000 \mathrm{~Hz}$. Horizontal and vertical movements of the right eye were recorded by means of an infrared video-based eye tracker (Desktop Eyelink 1000 system; SR Research) at a sampling rate of $1000 \mathrm{~Hz}$. A calibration was performed before every block of trials by means of a fixation grid composed of 9 known locations.

A

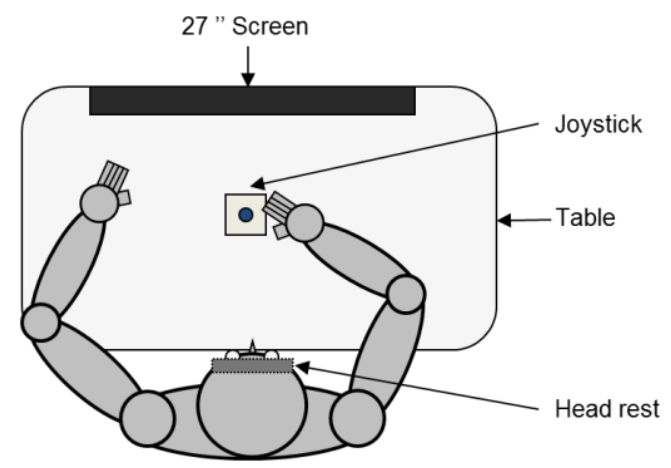

B

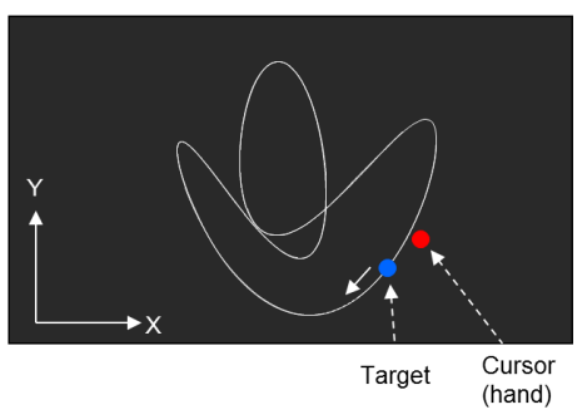


Fig. 1- Apparatus and experimental task. A. Top view of a participant sitting in the experimental setup. B. Schematic view of the screen during hand tracking. The target path was not displayed on the screen (see Methods for further information).

\subsection{Experimental Design}

The tracking task (see Fig. 1B) consisted of moving the joystick with the right hand so as to keep the cursor (red disk, $0.5^{\circ}$ in diameter) as close as possible to the moving target (blue disk, $0.5^{\circ}$ in diameter). The gain of the joystick was such that a $25^{\circ}$ change in joystick angle resulted in a $15 \mathrm{~cm}$ cursor displacement on the screen (this gain felt comfortable and made it impossible to move the cursor outside the screen). The delay between the joystick (hand) moving and the cursor doing so was only slightly longer than the time needed to render and display the cursor on the screen (on the order of 7-8 $\mathrm{ms}$ ). For the analysis, we synchronized the hand and gaze movements with the time at which the target was rendered, rather than the time at which the target was presented, so the cursor is synchronized with the target. Since we describe movements of the hand in cursor coordinates we refer to them as cursor movements, although their timing actually corresponds with that of the hand rather than of the cursor. The advantage of doing so is that eye and hand movements remain synchronized.

The tracking task allowed us to probe the ability to master hand movement along a desired trajectory (Ogawa \& Imamizu, 2013; Tong \& Flanagan, 2003). The motion of the target resulted from a combination of sinusoids: two along the frontal axis, and two on the sagittal axis. The following equations determined the target's motion:

$$
\begin{aligned}
& x_{t}=A_{1 x} \cos \omega t+A_{2 x} \cos \left(h_{x} \omega t-\varphi_{x}\right) \\
& y_{t}=A_{1 y} \sin \omega t+A_{2 y} \sin \left(h_{y} \omega t-\varphi_{y}\right)
\end{aligned}
$$

This technique was used to generate pseudo-random 2D patterns while preserving smooth changes in velocity and direction (Danion \& Flanagan, 2018; Mrotek \& Soechting, 2007; Soechting et al., 2010). A total of 5 different patterns were used throughout the 
experiment, each with a mean tangential velocity of $16 \mathrm{~cm} / \mathrm{sec}$ (see Table 1 and Fig. 2). Each trial had a duration of $10 \mathrm{~s}$. Given that the time necessary to complete a full revolution was $5 \mathrm{~s}$, each movement pattern was repeated twice per trial. The order in which the patterns were presented was randomized across trials while making sure that each pattern was presented twice (but never consecutively). Due to an error, one participant performed the correct number of trials but did not perform each pattern twice, but since each pattern was presented at least once the data were included in the analysis.

\begin{tabular}{ccccccccc} 
Trajectory & $A_{1 x}(\mathrm{~cm})$ & $A_{2 x}(\mathrm{~cm})$ & $\mathrm{h}_{\mathrm{x}}$ & $\varphi_{\mathrm{x}}\left({ }^{\circ}\right)$ & $A_{1 y}(\mathrm{~cm})$ & $A_{2 y}(\mathrm{~cm})$ & $\mathrm{h}_{\mathrm{y}}$ & $\varphi_{\mathrm{y}}\left({ }^{\circ}\right)$ \\
\hline 1 & 5 & 5 & 2 & 45 & 5 & 5 & 3 & -135 \\
2 & 4 & 5 & 2 & -60 & 3 & 5 & 3 & -135 \\
3 & 4 & 5.1 & 3 & -60 & 4 & 5.2 & 2 & -135 \\
4 & 5 & 5 & 3 & 90 & 3.4 & 5 & 2 & 45 \\
5 & 5.1 & 5.2 & 2 & -90 & 4 & 5 & 3 & 22.5
\end{tabular}

Table 1. Target trajectory parameters.

As already mentioned, the data were taken from the initial baseline trials of several experiments (Gouirand et al., 2019; Mathew et al., 2018, 2019). Although these experiments were carried out with different objectives in mind, and different conditions after the baseline, they all started with a block of 10 trials to assess the baseline performance of each participant. The current study uses the data collected during these baseline trials. During the baseline trials, the tracking task was performed with the conventional mapping between the joystick and cursor motion (right=right, left=left, forward=upward, backward=downward). Prior to the experiment, each participant performed 2 or 3 practice trials to become familiarized with the setup and the tracking task. 


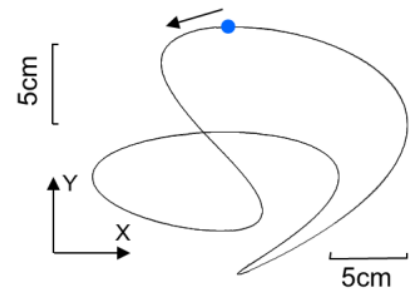

Pattern 1

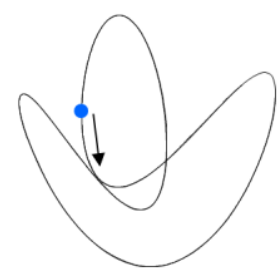

Pattern 2

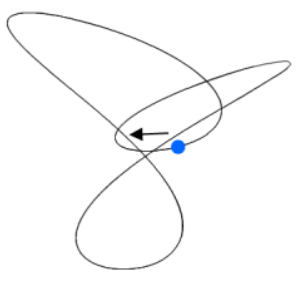

Pattern 3

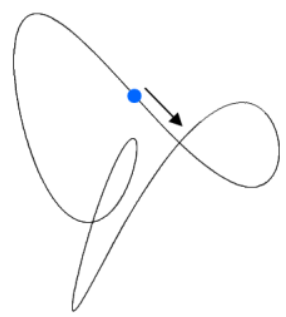

Pattern 4

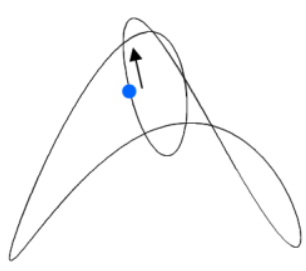

Pattern 5

Fig. 2- The five target patterns employed for manual tracking (see Methods for further information).

\subsection{Data Analysis}

Our main goal was to assess asymmetries between horizontal and vertical tracking, both in eye movements and in hand movements. To quantify how well participants tracked the target with their gaze, we measured the horizontal and vertical distance between gaze and target at each instant, and then averaged this distance across time for each trial. We considered all measurements (at $1000 \mathrm{~Hz}$ ) from $1 \mathrm{~s}$ after the movement started until it ended, which gave us up to 9000 data points per direction per trial. Although we measured eye and cursor (hand or joystick) movements at $1000 \mathrm{~Hz}$, the images of the target and the cursor were only presented at the frame rate of the screen $(144 \mathrm{~Hz})$. We used the target's position according to the equations that were used to generate the target motion to generate values at $1000 \mathrm{~Hz}$. To quantify how well participants tracked the target with their hand, we did the same thing for the distance between the cursor (moved by the hand) and the target. We also determined the distance between gaze and cursor to judge to what extent they tracked the target with their eyes and hand together. We determined whether the distances differed in a consistent manner between the two directions, for all target trajectories, with a repeated 
measure ANOVA on the mean distances, with the within-participant factors direction (horizontal or vertical) and trajectory.

There are several possible reasons why tracking precision may differ in the horizontal and vertical directions, both for gaze and for the cursor (hand). If the ability to anticipate the target's motion differs, or if the ability to move the eyes and cursor to follow the target differs, lags will also differ. Since a longer lag would almost automatically result in tracking the target at a larger distance, and possibly also less smoothly, we analyzed the lags and reexamined all the distances between gaze, cursor and target after correcting for any differences in lag. We then further examined differences in pursuit and in the contribution of saccades.

We analyzed potential differences in lag in two ways. Both started with determining to what extent gaze (or cursor) lags behind or anticipates the target's motion. We did this separately for the horizontal and vertical components of tracking. This was achieved by finding the peak in the cross-correlation between the two time series involved. We used a repeated measure ANOVA to compare the fit horizontal and vertical lags across participants and trajectories. This examines whether there is consistency in the difference between lags across participants, and whether such a difference could be due to the specific trajectory used. The trajectories were reasonably well-matched in difficulty in the two directions, but they were not identical in both directions, so we considered this to be worthwhile checking. Since each participant was exposed to each trajectory twice, we averaged the lags across these two trials before conducting the ANOVA. We also determined the correlation between the lags for the horizontal and vertical direction for both gaze and cursor, and the correlation between the lags for gaze and cursor in the two directions (across participants after averaging across trajectories).

In order to get a feeling for how consistently participants tracked better in the horizontal than the vertical direction, in addition to the above-mentioned comparison of the 
differences between the lags in the two directions, we also examined the extent to which it was justified to fit two separate lags. To do so, we used sum-of-squares F tests to compare the fits of three nested models. We conducted this analysis for individual trials and report the number of trials for which the comparisons are significant at the $1 \%$ level. The three models that we consider are one in which there are different lags for the horizontal and vertical direction, one in which there is a single lag for both directions, and one in which there is no lag at all. For the first model, we determine the lag separately for the horizontal and vertical components of the time series, as described above. We then sum the squares of the differences between the measured positions of gaze (or cursor) and the shifted target position across the points for each direction. For the second model, we determine the lag in the same manner, but searching for the peak in the sum of the cross-correlation across the two directions simultaneously to obtain a single lag (see Danion and Flanagan, 2018). We then determine the sum-of-squares in the same manner as when considering separate lags. Finally, for the third model, we determine the sum-of-squares in the same manner without shifting any of the time series.

We then performed two tests. The first determined whether fitting a lag was justified at all and the second determined whether we can better fit two lags than one. Both tests involved comparing nested models, so we used F-tests to determine whether the improvement that is achieved by adding a fit parameter is large enough to justify adding the parameter. We determined a normalized measure of the decrease in variability due to the fit parameter (a lag) by dividing the decrease in the sum-of-squares when adding the fit parameter by the sum-ofsquares of the model with the additional parameter. The analysis is complicated by the fact that there are different numbers of points to consider in different cases because the number of points for which we have a measurement and a target position depends on the lag (and on missing data, for instance due to blinks). We therefore normalized the sums of squares to the 
number of measurements before determining the relative change, and used the smallest number of measurements to determine the related change in degrees of freedom (one divided by the smallest number of measurements minus one). If we divide the value of the former by the latter we obtain an F-value. For one parameter and more than 1000 points an F-value larger than 6.66 indicates that adding the parameter improves the fit significantly at the $1 \%$ level. We only consider the fit to be better if adding a parameter improves the fit significantly at this level.

We also determined to what extent residual errors for gaze and cursor are correlated, and to what extent participants who tracked better with gaze also did so with the cursor. Since the former correlation could just be a consequence of the target trajectory, and the latter could just be a consequence of some participants taking the experiment more seriously, we also conducted a more elaborate analysis of the eye-cursor distance. During each 10s trial, the pattern of target motion was repeated twice: 2 loops of $5 \mathrm{~s}$ each. We used this to compute the mean eye-cursor distance in two ways. First, we computed this distance over the last 4 seconds of each loop and averaged the values (NORMAL). Second, we computed this distance in the same manner after switching the hand movements across the 2 loops, so after aligning the eye data of the first loop with the cursor data of the second loop, and aligning the eye data of the second loop with the cursor data of the first loop (SWITCHED). We reasoned that if the hand and eye track the target independently of each other, these two computations should give a similar result, even if some errors are caused by characteristics of the target's path, and even if some participants generally track better than others, because both the trajectory and the participant are the same in both cases. If eye and hand do not follow the target independently, the NORMAL distance will be smaller than the SWITCHED distance.

Finally, for gaze, we examined whether any difference in lag between the horizontal and vertical directions is related to making saccades. A difference in lag might arise if 
saccades are initiated when the target reaches a different retinal eccentricity in the horizontal and vertical direction. Conversely, making more saccades may decrease the lag. We therefore separated periods of smooth pursuit, saccades, and blinks from the raw eye position signals (Mathew et al., 2018) and evaluated the gain of smooth pursuit by averaging the ratio between instantaneous gaze and target tangential velocities (at times at which the target was moving at more than $10 \mathrm{~cm} / \mathrm{s}$; see Landelle et al. 2016). This was done separately for the horizontal and vertical components of the eye movements. We also determined the relationship between the directions of saccades, their amplitudes, and the eccentricities of the targets when the saccades were initiated.

\section{Results}

\subsection{Representative trial}

Figure 3 shows one representative trial. In this example, vertical tracking looks somewhat poorer than horizontal tracking both for the eye and the hand. In the next sections this observation will be analyzed in more detail. 

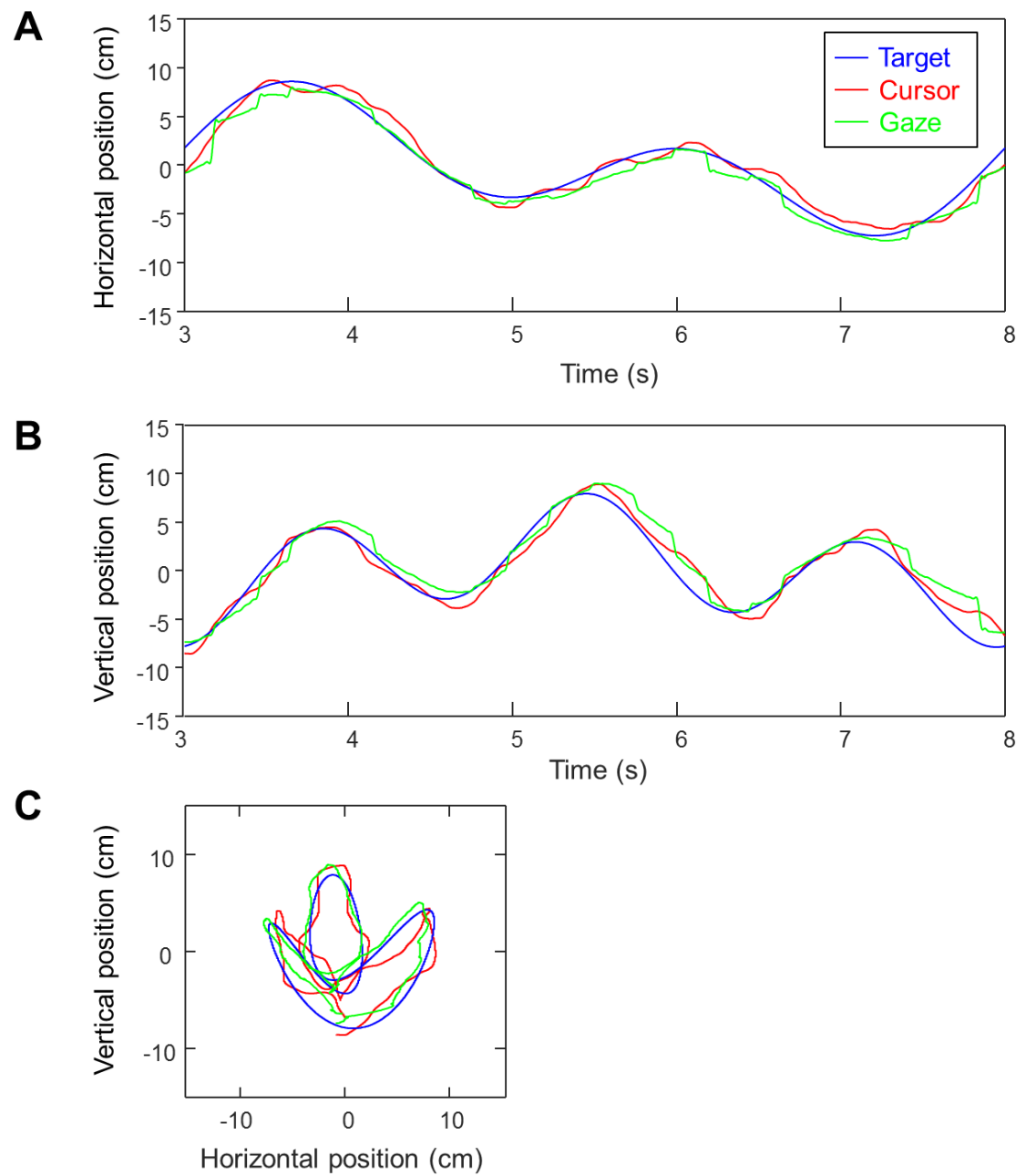

Fig. 3- A typical example of how the eye and hand track an unpredictably moving target. A. Horizontal components of gaze (eye), cursor (hand) and target motion. B. Same for vertical components. C. Corresponding spatial trajectories. Note the somewhat greater accuracy of horizontal tracking. 
A

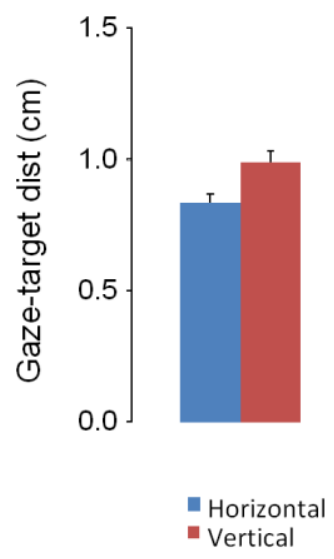

D

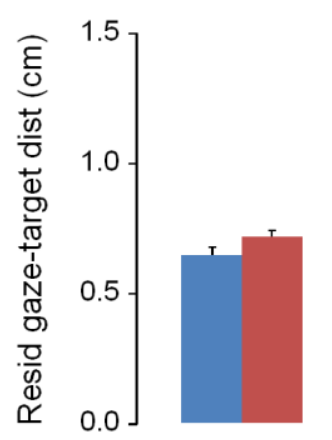

B

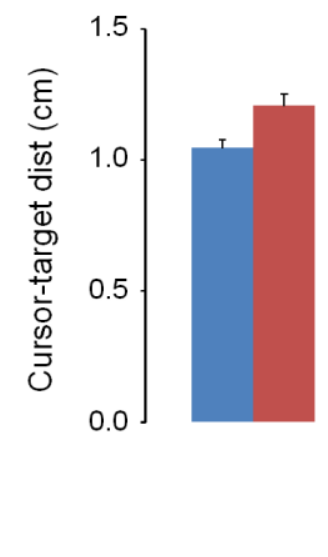

E

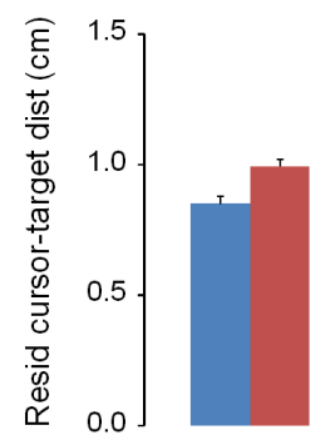

C

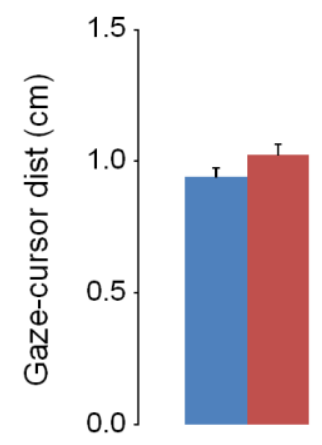

$\mathbf{F}$

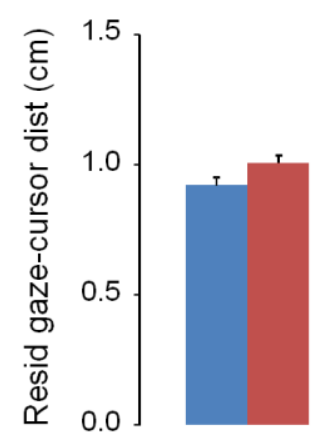

Fig. 4- Horizontal and vertical tracking performance for gaze and cursor. All values are mean distances. A-C. Actual distances. D-F. Distances after correcting for the fit lags. Note that all the horizontal distances are slightly smaller than the vertical distances, both before and after the correction.

\subsection{Eye tracking performance}

Eye tracking of the target was typically more accurate in the horizontal direction than in the vertical one. The mean absolute distance between gaze and target was $0.84 \mathrm{~cm}$ in the horizontal direction and $0.99 \mathrm{~cm}$ in the vertical direction $(\mathrm{F}(1,61)=18.42 ; \mathrm{p}<0.001$; repeated measures ANOVA across participants' mean values; Fig. 4A). Part of this difference might arise from differences in the extent to which gaze lags the target in the two directions, because nested F-tests revealed that 466 of the 620 trials (10 for each of the 62 participants) were fit best using two lags (75\%). The remaining 154 were fit best with one lag (i.e. the same lag in both directions). None were fit best with no lag. On average, the lag between target and gaze was $8 \mathrm{~ms}$ longer for the vertical component than for the horizontal one $(F(1,61)=44.8$; 
$\mathrm{p}<0.0001$; see central part of Fig. 5A). The lag also differed between patterns $(\mathrm{F}(4,244)=9.0$; $\mathrm{p}<0.0001)$ and there was a significant interaction between direction and pattern $(\mathrm{F}(4,244)=23.5 ; \mathrm{p}<0.0001)$. Bonferroni corrected post-hoc tests revealed that the lag was significantly larger $(\mathrm{p}<0.05)$ for pattern 3 than for the other patterns. The differences in lag between participants' horizontal and vertical tracking were considerably smaller than the differences in lag between participants: the horizontal and vertical lags were highly correlated across participants (green dots in Fig. 5B; Pearson's product-moment correlation $\mathrm{r}=0.81$ ). Thus, if a participant was slow in one direction, he or she was likely to also be slow in the other direction.
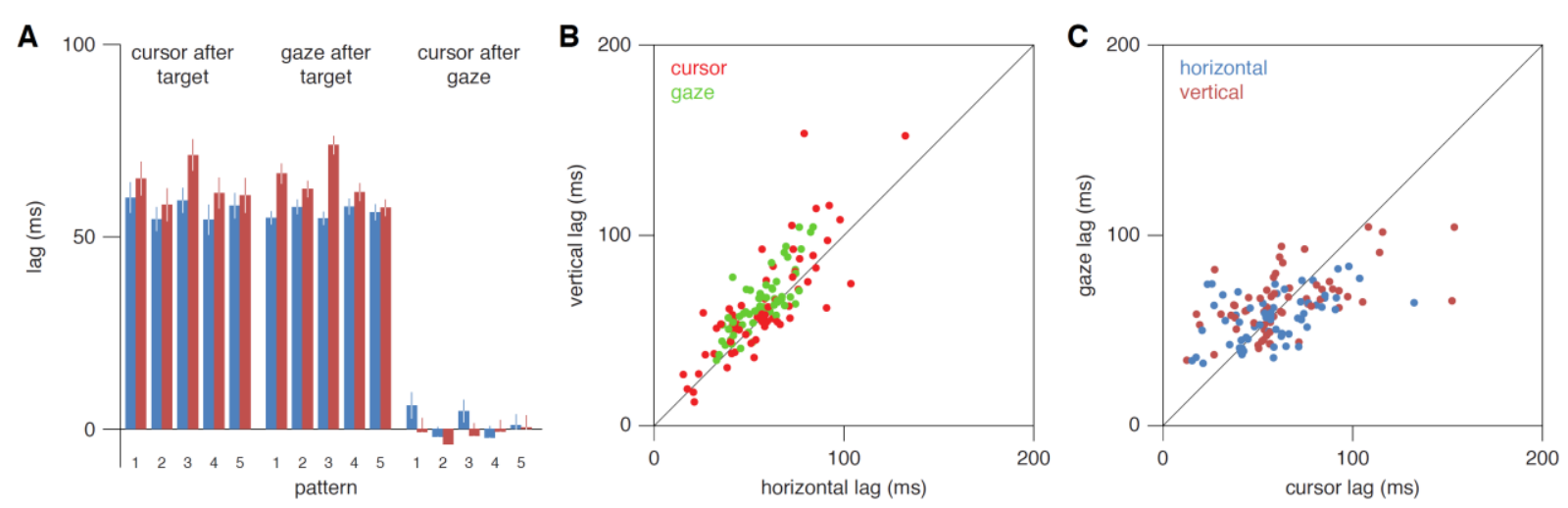

Fig. 5- Summary of the lag data. A. The mean lag was longer for the vertical component of the target's motion (red) than for the horizontal component (blue). It was quite similar for the cursor and gaze, both of which lagged slightly more than $50 \mathrm{~ms}$ after the target. There were some differences in lag between the different patterns of target motion but the asymmetry between horizontal and vertical was present for all patterns. B. Correlation between the lags for the horizontal and vertical component of target motion. Each point represents one participant's mean value across the five patterns (10 trials). Each participant is represented by two dots: one for movements of the hand (cursor; red) and the other for movements of the eyes (gaze; green). C. Correlation between the lags of the eyes (gaze) and hand (cursor). Again, each participant is represented by two dots: one for the horizontal component of the tracking behavior (blue) and the other for the vertical component (red).

Computing the mean absolute distance between gaze and target after shifting the data to account for the two fit lags per trial reduced the value from 0.84 to $0.65 \mathrm{~cm}$ for the horizontal direction, and from 0.99 to $0.76 \mathrm{~cm}$ for the vertical direction (Fig. 4D). Thus, eye tracking errors only decreased from being a bit more than $18 \%$ larger in the vertical direction to being about $17 \%$ larger, indicating that the poorer eye tracking performance in the vertical 
direction is not only (or even mainly) caused by the longer lag. Analyzing smooth pursuit and saccades revealed further directional asymmetries. We found that the mean smooth pursuit gain was $8 \%$ lower in the vertical direction than in the horizontal one $(0.71$ and 0.77 , respectively; $\mathrm{F}(1,61)=18.6 ; \mathrm{p}<0.001)$, with the two gains being correlated across participants $(\mathrm{r}=0.71 ; \mathrm{p}<0.001)$. We also found that the positional error at catch-up saccade initiation was $19 \%$ larger in the vertical direction than in the horizontal one (1.46 and $1.23 \mathrm{~cm}$ respectively; $\mathrm{F}(1,61)=33.9 ; \mathrm{p}<0.001)$. This was not just because people tolerated larger vertical gazetracking errors than horizontal ones, because if that were the case we would expect smaller saccades for tracking errors in the horizontal direction. What we found is fewer saccades of all sizes when the tracking error was primarily horizontal (Fig. 6A). The catch-up saccades themselves were less clearly biased towards the vertical (Fig. 6B) but they were clearly directed towards the position of the target near saccade onset (Fig. 6C). The bias in direction to the target at saccade onset may therefore result from poorer vertical pursuit, rather than from tolerating larger vertical errors. The fact that saccade directions did not precisely match the target positions could be because the saccade is based on earlier information and because the direction of target motion is also considered.
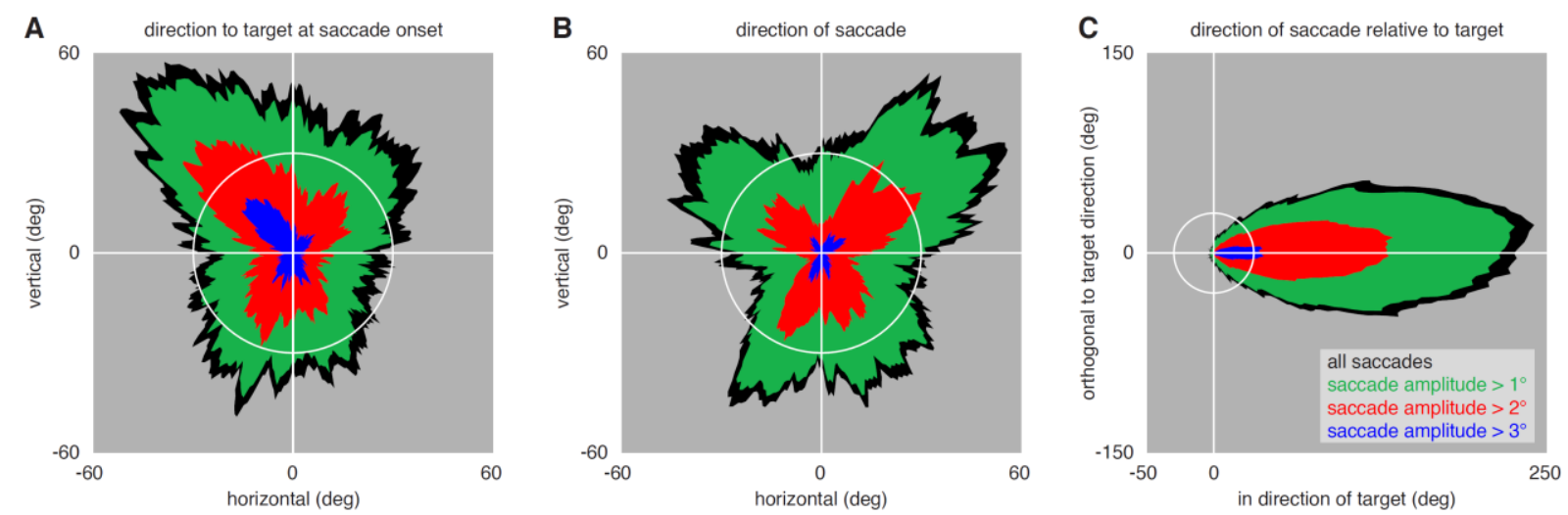

Fig. 6- Number of saccades for which various measures fall within $5^{\circ}$ of each direction. The white circle indicates 30 saccades. $\boldsymbol{A}$. The direction to the target at saccade onset $\boldsymbol{B}$. Directions of saccades. $\boldsymbol{C}$. Directions of saccades with respect to the target rather than in space. 
We also examined the mean horizontal and vertical distance between gaze and cursor, both before (Fig. 4C) and after (Fig. 4F) considering the fit lags. For the data before fitting any lags, a two-way ANOVA revealed that the gaze-target distance was smaller than the gazecursor distance $(\mathrm{F}(1,61)=14.28 ; \mathrm{p}<0.001)$, confirming that gaze is directed at the target rather than the cursor (Danion \& Flanagan, 2018). The distances were also smaller in the horizontal direction than in the vertical direction $(\mathrm{F}(1,61)=15.15 ; \mathrm{p}<0.001)$, with a significant interaction between kind of distance and direction indicating that the directional asymmetry is smaller in the gaze-cursor distance than in the gaze-target distance $(F(1,61)=10.32 ; \mathrm{p}<0.01)$.

\subsection{Hand tracking performance}

Manual tracking was also more accurate in the horizontal than the vertical direction. The mean absolute distance between the cursor and target was $1.05 \mathrm{~cm}$ in the horizontal direction and $1.21 \mathrm{~cm}$ in the vertical direction $(\mathrm{F}(1,61)=52.8 ; \mathrm{p}<0.001$; Fig. 4B $)$. As discussed earlier for eye tracking, part of the difference might originate in differences in the extent to which the hand lags the target in the two directions. Nested F-tests show that 540 of the 620 trials were fit best using two lags (87\%). Of the remaining tests, 79 were fit best with one lag and for one there was no need to fit a lag. On average, the lag between target and cursor was 6 ms longer for the vertical component than for the horizontal one $(F(1,61)=8.5 ; p=0.005$; see left part of Fig. 5A). The lag also differed between patterns $(F(4,244)=3.9 ; p=0.004)$, with no significant interaction $(\mathrm{F}(4,244)=1.2 ; \mathrm{p}=0.3)$. Bonferroni corrected post-hoc tests revealed that the lag was significantly larger for pattern 3 than for patterns 2 and 4. Again, the horizontal and vertical lags were highly correlated across participants (red dots in Fig. 5B; r=0.82).

Computing the mean absolute distance between the cursor and target after shifting the data to account for the two fit lags per trial reduced the value from 1.05 to $0.85 \mathrm{~cm}$ for the horizontal direction, and from 1.21 to $0.99 \mathrm{~cm}$ for the vertical direction (Fig. 4E). Thus, 
manual tracking errors increased from being almost $16 \%$ larger in the vertical direction to being about $17 \%$ larger, indicating that the longer lag is not responsible for the poorer tracking performance in the vertical direction.

\subsection{Eye-Hand coordination}

Across participants, the lag of the cursor was correlated with the lag of gaze, both in the horizontal direction (blue dots in Fig. 5C; $r=0.48$ ) and in the vertical direction (red dots; $\mathrm{r}=0.57$ ). The lags were not only correlated, but were also quite similar in magnitude, so the lag between gaze and cursor was negligible (right part of Fig. 5A).

When analyzing the distances between gaze, cursor and target we used the actual values, without considering the lags. Although the gaze-cursor distance (Fig. 4C) was not particularly small, showing that gaze was not tracking in the same way as the cursor, within individual participants' data the tracking errors of gaze and cursor were correlated (mean across trials and participants of $r=0.60$ for the horizontal direction and $r=0.62$ for the vertical direction). Moreover, participants who tracked the target better with their gaze also tended to track it better with the cursor (correlation across participants between the mean target-cursor distance and the mean target-gaze distance: $\mathrm{r}=0.65)$.

Comparing the mean gaze-cursor distance in the NORMAL and SWITCHED traces (see Methods for details) shows that the eye and hand do not follow the target independently. The mean gaze-cursor distance was $1.54 \mathrm{~cm}$ for the NORMAL traces and $1.65 \mathrm{~cm}$ for the SWITCHED traces $(\mathrm{F}(1,61)=191.3, \mathrm{p}<0.001)$. The average difference per participant is $7.8 \%$ with a $95 \%$ confidence interval of $6.8 \%-8.7 \%$. This difference does not simply arise because participants can better anticipate the trajectory when it is repeated, because although the mean target-cursor distance improved by about 7\% when the trajectory was repeated (from $1.82 \mathrm{~cm}$ during the first loop to $1.70 \mathrm{~cm}$ during the second loop), there was clearly no such 
improvement for the target-gaze distance $(1.41 \mathrm{~cm}$ during the first loop; $1.44 \mathrm{~cm}$ during the second loop).

\section{Discussion}

Our main objective was to investigate the possible asymmetry between vertical and horizontal tracking with the hand and eyes during manual tracking. Our results can be summarized with the following key findings. First, we observed that both the eye and hand consistently tracked the target more precisely along the horizontal axis than along the vertical one. Second, significantly smaller residual errors were obtained when considering separate lags for horizontal and vertical tracking than when considering a single lag for both directions. Third, for both effectors the lag with respect to the target was shorter in the horizontal direction than the vertical one. Fourth, compensating for the longer lag of vertical eye and hand tracking did not get rid of the directional asymmetry in tracking precision. Fifth, despite the presence of a directional asymmetry, the lags of vertical and horizontal tracking were correlated across participants. Sixth, the mean lags of the eye and hand were nearly identical, and somewhat correlated across participants. Finally, the errors in tracking the target were fairly related for the hand and eye. We now discuss these findings and their implications in more detail.

\subsection{Directional asymmetry in gaze behaviour}

We consistently found that the eye was almost $20 \%$ closer to the target horizontally than vertically. Our participants did not receive explicit instructions to follow the targets with their eyes. Nevertheless, the asymmetry was similar to that found in previous studies in which participants were explicitly asked to follow the targets with their eyes (Baloh et al., 1988; Collewijn \& Tamminga, 1984; Grönqvist et al., 2006; Ke et al., 2013; Lipton et al., 1980; Robert et al., 2014; Rottach et al., 1996; Takeichi et al., 2003). Our results also nicely 
complement earlier observation that smooth pursuit gain is higher in the horizontal direction (Collewijn \& Tamminga, 1984; Ke et al., 2013; Mrotek \& Soechting, 2007).

In this study we also found asymmetries in saccades. We observed that the gaze-target distance was larger when initiating vertical catch-up saccades as compared to horizontal ones. We also found that fewer saccades were made in response to horizontal than vertical tracking errors. This combination of findings suggests that the larger gaze-target distance at saccade onset results from poorer vertical pursuit, rather than from tolerating larger vertical errors, because otherwise we would have expected fewer (but larger) vertical than horizontal saccades. Importantly, the effects that we report are unlikely to result from imperfections in our technique for recording eye movements or from differences between the way in which horizontal and vertical eye movements are controlled because the asymmetry was very similar (and correlated) for movements of the hand, that was recorded using very different technology (the joystick) and activated with a completely unrelated set of muscles.

\subsection{Directional asymmetry in hand tracking}

We show that the directional asymmetry in gaze tracking is accompanied by a similar asymmetry in hand tracking. The magnitude of the effect is even rather similar for eye and hand movements. Not only was hand tracking error $16 \%$ greater for vertical hand movements ( $18 \%$ for eye movements), but those movements lagged the target to a $6 \mathrm{~ms}$ larger extent (this was $8 \mathrm{~ms}$ for eye movements). To our knowledge, such directional asymmetry in hand tracking has never been reported before.

Inertial and gravitational forces can influence hand movement kinematics differently in different directions (Crevecoeur et al., 2009; Flanagan \& Lolley, 2001; Papaxanthis et al., 1998). However, here the hand movements that were performed with the joystick were small $(<5 \mathrm{~cm})$. Moreover, they were performed in the horizontal plane and the forearm was 
supported. Anatomical wrist or finger muscle characteristics could provide an advantage for horizontal cursor movement, for instance by involving pronators and supinator's rather than flexors and extensors, but we consider it unlikely that this could account for the asymmetry because participants did not necessarily all hold the joystick in the same way. Overall we conclude that neither the musculo-skeletal design of the hand, nor the hand controller, are likely to be key factors in the directional asymmetry of manual tracking.

\subsection{Eye-hand coordination and directional asymmetry}

Despite the many similarities and correlations that we found between tracking with the eye and tracking with the hand, the distance between gaze and cursor was not particularly small. This might appear to contradict our claim that the eye and hand are guided by a common prediction of target motion, but the different biomechanical properties of the eye and hand can give rise to quite different trajectories even if both eye and hand are based on the same information. The hand has both larger inertia and is located further away from neural control centres than the eye, so one expects it to have a longer motor delay. The similar fit lag for the two effectors at the behavioural level (Fig. 5A) therefore suggests that hand movements are initiated earlier than eye movements. There was no explicit requirement to synchronize eye and hand movements, but the cerebellum, being a key structure for eye-hand coordination (Gauthier et al., 1988; Miall et al., 2001) as well as for movement synchronization across effectors (Cerri et al., 2005), might compensate for the different mechanical delays by predicting further into the future for the hand. Doing so will obviously introduce differences in errors between gaze and hand. Moreover, the eyes can make fast, large saccades to correct for large errors. The hand must adjust more gradually. The different errors for gaze and cursor that arise from such factors are quickly amplified, because once the required corrections are different, the adjustments and the errors in adjustments are too. We 
therefore wish to emphasize that despite differences in biomechanics and corrective mechanisms, the eye and hand clearly do not track the target independently. Our analysis of the gaze-cursor distance using positions from the same or different loops of the same trajectory provides compelling evidence that the two effectors share some common input.

While tracking the target, participants were presumably constantly predicting how it would continue to move in the near future so that they could incorporate such predictions within the ongoing hand and eye movements. The fact that we observed gaze-target and cursor-target lags on the order of 50-60 ms suggests that predictive processes must be involved, because otherwise, the lag would be at least 100ms (Brenner \& Smeets, 1997; Mathew et al., 2019). If we consider the lag as a compromise between following the target and predicting how it will move, we can interpret the directional asymmetry in tracking the target (both with gaze and with the cursor) as the predictive process having a stronger influence for horizontal than for vertical motion.

We propose that more precise prediction of horizontal motion allows participants to rely more on prediction, which gives rise to a smaller lag. It is possible that there is some other reason for predicting further into the future for horizontal motion, and that doing so gives rise to a higher precision for tracking horizontal motion. We feel it is more likely that a difference in precision is the origin rather than a consequence, because the horizontal motion that we experience in daily life is normally close to constant, whereas it is not uncommon to encounter vertical acceleration caused by gravity. Considering gravitational acceleration (Jörges \& López-Moliner, 2019) is likely to reduce one's confidence in predicting vertical motion. This reasoning is based on the assumption that the extent to which one relies on predicted motion depends on the precision of the prediction, which is consistent with optimizing the use of information (because the more reliable the prediction the less likely it is 
that using it will increase rather than decrease errors) but for which we have no direct evidence.

The main contribution of this work is to reveal a directional asymmetry that is shared between eye and hand movements when both effectors are simultaneously engaged in tracking a moving target. Directional asymmetries in eye movements and hand movements have been reported in separate studies, but to our knowledge, our study is the first to provide evidence for a directional asymmetry that transcends both effectors, which further elucidates the intricate relationship between eye and hand movements during visually guided actions (Crawford et al., 2004; de Brouwer et al., 2018; Johansson et al., 2001). Although manual tracking relies more on feedback loops than reaching (Yang et al., 2020), so prediction may play a different role in the two kinds of movements, there is also some evidence that (attentional) processing is shared between the eye and hand in reaching (Khan et al., 2011; Neggers \& Bekkering, 2000; but see Hanning et al., 2018; Jonikaitis \& Deubel, 2011).

\subsection{Possible origins of directional bias, and limitations of the study}

The observation that horizontal eye movements are consistently more accurate than vertical ones has led to several propositions. A main one is that "in daily life most objects which are pursued move in a more or less horizontal plane (the predominant direction of locomotion and traffic) and subjects get an everyday training in horizontal pursuit" (Collewijn \& Tamminga, 1984). Although this reasoning holds for eye movements by a static observer, it is unclear how it can account for biases in hand movements. It is also not necessarily true when maintaining gaze on static objects while walking, so it would only apply to the pursuit of moving objects. It would be interesting to see whether the same asymmetry is also present when the observer is moving rather than the target. Studies on optokinetic nystagmus (Hainline et al., 1984; Knapp et al., 2013) suggest that there may be differences, but it is not clear whether such differences are due to differences in anticipating the 'target' motion. 
In the current study, we speak about horizontal and vertical motion but for the hand the motion is never vertical. It is the cursor's motion in response to the hand movement that is vertical. The hand movement is also 'vertical' in terms of predicting the target's motion. We did not vary participants' head orientation so we do not know whether the asymmetry follows the orientation of the body, with directions being related to the orientation of the head and 'horizontal' corresponding to the inter-ocular axis, or whether the asymmetry is related to space, with 'vertical' corresponding to the direction of gravity. Although we do not know the origin of the directional asymmetry favouring horizontal movements, this effect should certainly be considered in studies that investigate the accuracy of visually guided hand movements by means of a joystick and a computer screen. If we are correct in attributing the asymmetry to differences in prediction, the asymmetry should also be considered when using any other device to track a target by moving a cursor across a screen.

Since the patterns were not specifically designed for this study, the horizontal and vertical components of the target's motion were not precisely equated across the five patterns. The fact that we found an influence of pattern on the lag, both for gaze and hand, and even an interaction between direction and pattern for the lag of gaze, shows that the lag depends on the tracking trajectory. We checked that the dependence on direction is not too obviously a result of the specific target motion in these trajectories by verifying that they were all of similar complexity in the vertical and horizontal directions. To do so, we estimated the predictability (using approximate entropy, see Mathew, Sarlegna, et al., 2019; Pincus, 1991) as well as the mean and standard deviation of the horizontal and vertical speed. ANOVAs did not reveal consistent directional effects across the five patterns for any of these measures $(\mathrm{F}(1,4)<0.535, \mathrm{p}>0.5)$, suggesting that overall our target trajectories did not particularly favor vertical or horizontal movements. Most importantly, although there were significant differences in the magnitude of both the delay itself and the difference in delay across the five patterns, the tendency for the lag to be longer in the vertical direction was visible for each of 
the patterns (Fig. 5A). Thus, we consider it unlikely that our findings are simply the result of an unfortunate choice of target paths.

\subsection{Concluding comments}

Our observations provide further support for an intricate relationship between eye and hand movements (Carey, 2000; Crawford et al., 2004; Johansson et al., 2001; Land \& McLeod, 2000; Li et al., 2018; Miall et al., 2001), in the sense that a similar directional asymmetry was observed for these two effectors. More specifically these results suggest that when tracking a moving target, predictive processes linking ongoing target motion with forthcoming hand and eye actions are shared across effectors. It remains unclear why those processes are worse for vertical than horizontal motion, but our guess is that poorer predictions in the vertical direction give rise to longer lags because one predicts less far into the future, so that pursuit has a lower gain and is less smooth (more saccades), and tracking errors are larger. 


\section{REFERENCES}

Baloh, R. W., Yee, R. D., Honrubia, V., \& Jacobson, K. (1988). A comparison of the dynamics of horizontal and vertical smooth pursuit in normal human subjects. Aviation, Space, and Environmental Medicine, 59(2), 121-124.

Bar, M. (2007). The proactive brain : Using analogies and associations to generate predictions. Trends in cognitive sciences, 11(7), 280-289. https://doi.org/10.1016/j.tics.2007.05.005

Barnes, G. R. (2008). Cognitive processes involved in smooth pursuit eye movements. Brain and Cognition, 68(3), 309-326. https://doi.org/10.1016/j.bandc.2008.08.020

Bock, O. (1987). Coordination of arm and eye movements in tracking of sinusoidally moving targets. Behavioural Brain Research, 24, 93-100.

Brenner, E., \& Smeets, J. B. (1997). Fast Responses of the Human Hand to Changes in Target Position. Journal of Motor Behavior, 29(4), 297-310. https://doi.org/10.1080/00222899709600017

Bubic, A., von Cramon, D. Y., \& Schubotz, R. I. (2010). Prediction, cognition and the brain. Frontiers in Human Neuroscience, 4, 25. https://doi.org/10.3389/fnhum.2010.00025

Carey, D. P. (2000). Eye-hand coordination : Eye to hand or hand to eye? Current Biology: $C B, 10(11), \mathrm{R} 416-419$.

Cerri, G., Esposti, R., Locatelli, M., \& Cavallari, P. (2005). Coupling of hand and foot voluntary oscillations in patients suffering cerebellar ataxia : Different effect of lateral or medial lesions on coordination. In Progress in Brain Research (Vol. 148, p. 227-241). Elsevier. https://doi.org/10.1016/S0079-6123(04)48019-4

Chubb, M. C., Fuchs, A. F., \& Scudder, C. A. (1984). Neuron activity in monkey vestibular nuclei during vertical vestibular stimulation and eye movements. Journal of Neurophysiology, 52(4), 724-742. https://doi.org/10.1152/jn.1984.52.4.724 
Clark, A. (2013). Whatever next? Predictive brains, situated agents, and the future of cognitive science. The Behavioral and Brain Sciences, 36(3), 181-204. https://doi.org/10.1017/S0140525X12000477

Collewijn, H., \& Tamminga, E. P. (1984). Human smooth and saccadic eye movements during voluntary pursuit of different target motions on different backgrounds. The Journal of Physiology, 351, 217-250.

Crawford, J. D., Medendorp, W. P., \& Marotta, J. J. (2004). Spatial transformations for eyehand coordination. Journal of Neurophysiology, 92(1), 10-19. https://doi.org/10.1152/jn.00117.2004

Crevecoeur, F., Thonnard, J.-L., \& Lefèvre, P. (2009). Optimal integration of gravity in trajectory planning of vertical pointing movements. Journal of Neurophysiology, 102(2), 786-796. https://doi.org/10.1152/jn.00113.2009

Danion, F. R., \& Flanagan, J. R. (2018). Different gaze strategies during eye versus hand tracking of a moving target. Scientific Reports, 8(1), 10059. https://doi.org/10.1038/s41598-018-28434-6

de Brouwer, A. J., Albaghdadi, M., Flanagan, J. R., \& Gallivan, J. P. (2018). Using Gaze Behavior to Parcellate the Explicit and Implicit Contributions to Visuomotor Learning. Journal of Neurophysiology. https://doi.org/10.1152/jn.00113.2018

Evinger, C., \& Fuchs, A. F. (1978). Saccadic, smooth pursuit, and optokinetic eye movements of the trained cat. The Journal of Physiology, 285, 209-229. https://doi.org/10.1113/jphysiol.1978.sp012568

Fiehler, K., Brenner, E., \& Spering, M. (2019). Prediction in goal-directed action. Journal of Vision, 19(9), 10. https://doi.org/10.1167/19.9.10 
Flanagan, J. R., \& Lolley, S. (2001). The inertial anisotropy of the arm is accurately predicted during movement planning. The Journal of Neuroscience: The Official Journal of the Society for Neuroscience, 21(4), 1361-1369.

Gauthier, G. M., Vercher, J. L., Mussa Ivaldi, F., \& Marchetti, E. (1988). Oculo-manual tracking of visual targets : Control learning, coordination control and coordination model. Experimental Brain Research, 73(1), 127-137.

Gouirand, N., Mathew, J., Brenner, E., \& Danion, F. (2019). Eye movements do not play an important role in the adaptation of hand tracking to a visuomotor rotation. Journal of Neurophysiology. https://doi.org/10.1152/jn.00814.2018

Grönqvist, H., Gredebäck, G., \& Hofsten, C. von. (2006). Developmental asymmetries between horizontal and vertical tracking. Vision Research, 46(11), 1754-1761. https://doi.org/10.1016/j.visres.2005.11.007

Hainline, L., Lemerise, E., Abramov, I., \& Turkel, J. (1984). Orientational asymmetries in small-field optokinetic nystagmus in human infants. Behavioural Brain Research, 13(3), 217-230. https://doi.org/10.1016/0166-4328(84)90164-5

Hanning, N. M., Aagten-Murphy, D., \& Deubel, H. (2018). Independent selection of eye and hand targets suggests effector-specific attentional mechanisms. Scientific Reports, 8(1), 9434. https://doi.org/10.1038/s41598-018-27723-4

Hogendoorn, H. (2020). Motion Extrapolation in Visual Processing : Lessons from 25 Years of Flash-Lag Debate. The Journal of Neuroscience: The Official Journal of the Society for Neuroscience, 40(30), 5698-5705. https://doi.org/10.1523/JNEUROSCI.027520.2020

Huang, C.-T., \& Hwang, I.-S. (2012). Eye-hand synergy and intermittent behaviors during target-directed tracking with visual and non-visual information. PloS One, 7(12), e51417. https://doi.org/10.1371/journal.pone.0051417 
Johansson, R. S., Westling, G., Bäckström, A., \& Flanagan, J. R. (2001). Eye-hand coordination in object manipulation. The Journal of Neuroscience, 21(17), 6917-6932. Jonikaitis, D., \& Deubel, H. (2011). Independent allocation of attention to eye and hand targets in coordinated eye-hand movements. Psychological Science, 22(3), 339-347. https://doi.org/10.1177/0956797610397666

Jörges, B., \& López-Moliner, J. (2019). Earth-Gravity Congruent Motion Facilitates Ocular Control for Pursuit of Parabolic Trajectories. Scientific Reports, 9(1), 14094. https://doi.org/10.1038/s41598-019-50512-6

Ke, S. R., Lam, J., Pai, D. K., \& Spering, M. (2013). Directional asymmetries in human smooth pursuit eye movements. Investigative Ophthalmology \& Visual Science, 54(6), 4409-4421. https://doi.org/10.1167/iovs.12-11369

Kettner, R. E., Leung, H. C., \& Peterson, B. W. (1996). Predictive smooth pursuit of complex two-dimensional trajectories in monkey : Component interactions. Experimental Brain Research, 108(2), 221-235.

Khan, A. Z., Song, J.-H., \& McPeek, R. M. (2011). The eye dominates in guiding attention during simultaneous eye and hand movements. Journal of Vision, 11(1), 9-9. https://doi.org/10.1167/11.1.9

Knapp, C. M., Proudlock, F. A., \& Gottlob, I. (2013). OKN asymmetry in human subjects : A literature review. Strabismus, 21(1), 37-49. https://doi.org/10.3109/09273972.2012.762532

Koken, P. W., \& Erkelens, C. J. (1992). Influences of hand movements on eye movements in tracking tasks in man. Experimental Brain Research, 88(3), 657-664.

Land, M. F., \& McLeod, P. (2000). From eye movements to actions : How batsmen hit the ball. Nature neuroscience, 3(12), 1340-1345. https://doi.org/10.1038/81887 
Landelle, C., Montagnini, A., Madelain, L., \& Danion, F. (2016). Eye tracking a self-moved target with complex hand-target dynamics. Journal of Neurophysiology, 116(4), 1859-1870. https://doi.org/10.1152/jn.00007.2016

Li, Y., Wang, Y., \& Cui, H. (2018). Eye-hand coordination during flexible manual interception of an abruptly appearing, moving target. Journal of Neurophysiology, 119(1), 221-234. https://doi.org/10.1152/jn.00476.2017

Lipton, R. B., Levin, S., \& Holzman, P. S. (1980). Horizontal and vertical pursuit eye movements, the oculocephalic reflex, and the functional psychoses. Psychiatry Research, 3(2), 193-203.

Mather, J. A., \& Putchat, C. (1983). Parallel ocular and manual tracking responses to a continuously moving visual target. Journal of Motor Behavior, 15(1), 29-38.

Mathew, J., Bernier, P.-M., \& Danion, F. R. (2018). Asymmetrical Relationship between Prediction and Control during Visuomotor Adaptation. ENeuro, 5(6). https://doi.org/10.1523/ENEURO.0280-18.2018

Mathew, J., Sarlegna, F. R., Bernier, P.-M., \& Danion, F. R. (2019). Handedness Matters for Motor Control But Not for Prediction. eNeuro, 6(3). https://doi.org/10.1523/ENEURO.0136-19.2019

Miall, R. C., Reckess, G. Z., \& Imamizu, H. (2001). The cerebellum coordinates eye and hand tracking movements. Nature Neuroscience, 4(6), 638-644. https://doi.org/10.1038/88465

Mrotek, L. A., \& Soechting, J. F. (2007). Target interception : Hand-eye coordination and strategies. The Journal of Neuroscience, 27(27), 7297-7309. https://doi.org/10.1523/JNEUROSCI.2046-07.2007

Neggers, S. F., \& Bekkering, H. (2000). Ocular gaze is anchored to the target of an ongoing pointing movement. Journal of Neurophysiology, 83(2), 639-651. 
Niehorster, D. C., Siu, W. W. F., \& Li, L. (2015). Manual tracking enhances smooth pursuit eye movements. Journal of Vision, 15(15), 11. https://doi.org/10.1167/15.15.11

Ogawa, K., \& Imamizu, H. (2013). Human sensorimotor cortex represents conflicting visuomotor mappings. The Journal of Neuroscience, 33(15), 6412-6422. https://doi.org/10.1523/JNEUROSCI.4661-12.2013

Papaxanthis, C., Pozzo, T., Vinter, A., \& Grishin, A. (1998). The representation of gravitational force during drawing movements of the arm. Experimental Brain Research, 120(2), 233-242. https://doi.org/10.1007/s002210050397

Pincus, S. M. (1991). Approximate entropy as a measure of system complexity. Proceedings of the National Academy of Sciences of the United States of America, 88(6), 2297-2301. https://doi.org/10.1073/pnas.88.6.2297

Prablanc, C., Echallier, J. F., Komilis, E., \& Jeannerod, M. (1979). Optimal response of eye and hand motor systems in pointing at a visual target. I. Spatio-temporal characteristics of eye and hand movements and their relationships when varying the amount of visual information. Biological Cybernetics, 35(2), 113-124.

Robert, M. P., Ingster-Moati, I., Albuisson, E., Cabrol, D., Golse, B., \& Vaivre-Douret, L. (2014). Vertical and horizontal smooth pursuit eye movements in children with developmental coordination disorder. Developmental Medicine and Child Neurology, 56(6), 595-600. https://doi.org/10.1111/dmcn. 12384

Rottach, K. G., Zivotofsky, A. Z., Das, V. E., Averbuch-Heller, L., Discenna, A. O., Poonyathalang, A., \& Leigh, R. J. (1996). Comparison of horizontal, vertical and diagonal smooth pursuit eye movements in normal human subjects. Vision Research, $36(14), 2189-2195$. 
Saito, Y., \& Sugimura, T. (2020). Different Activation Mechanisms of Excitatory Networks in the Rat Oculomotor Integrators for Vertical and Horizontal Gaze Holding. ENeuro, 7(1). https://doi.org/10.1523/ENEURO.0364-19.2019

Soechting, J. F., Rao, H. M., \& Juveli, J. Z. (2010). Incorporating prediction in models for two-dimensional smooth pursuit. PloS One, 5(9), e12574. https://doi.org/10.1371/journal.pone.0012574

Spering, M., Schütz, A. C., Braun, D. I., \& Gegenfurtner, K. R. (2011). Keep your eyes on the ball : Smooth pursuit eye movements enhance prediction of visual motion. Journal of Neurophysiology, 105(4), 1756-1767. https://doi.org/10.1152/jn.00344.2010

Takeichi, N., Fukushima, J., Kurkin, S., Yamanobe, T., Shinmei, Y., \& Fukushima, K. (2003). Directional asymmetry in smooth ocular tracking in the presence of visual background in young and adult primates. Experimental Brain Research, 149(3), 380-390. https://doi.org/10.1007/s00221-002-1367-3

Tong, C., \& Flanagan, J. R. (2003). Task-specific internal models for kinematic transformations. Journal of Neurophysiology, 90(2), 578-585. https://doi.org/10.1152/jn.01087.2002

Xia, R., \& Barnes, G. (1999). Oculomanual Coordination in Tracking of Pseudorandom Target Motion Stimuli. Journal of Motor Behavior, 31(1), 21-38.

Yang, C., Cowan, N., \& Haith, A. (2020). De novo learning versus adaptation of continuous control in a manual tracking task. bioRxiv. https://www.biorxiv.org/content/10.1101/2020.01.15.906545v1

Zhao, H., \& Warren, W. H. (2017). Intercepting a moving target : On-line or model-based control? Journal of Vision, 17(5), 12. https://doi.org/10.1167/17.5.12 